\title{
Adaptación Española de la Escala de Pasión al Ámbito Deportivo
}

\author{
Spanish Adaption of Passion Scale in Sport Context
}

\author{
Ignacio Pedrosa ${ }^{1}$, Eduardo García-Cueto ${ }^{2}$, Julio Torrado ${ }^{3}$ y Constantino Arce ${ }^{4}$
}

\section{Resumen}

La pasión experimentada hacia una actividad constituye uno de los principales factores explicativos del bienestar físico y emocional. El tipo de pasión desarrollada hacia el deporte, obsesiva o armoniosa, presenta diferentes consecuencias en términos emocionales y de adherencia. El presente trabajo plantea la adaptación al castellano de la Escala de Pasión en el contexto deportivo. En el primer estudio, la escala se aplicó a 146 deportistas $\left(\mathrm{M}_{\text {edad }}=15.94 ; \mathrm{DT}_{\text {edad }}=1.99\right)$. Una vez calibrada la escala, se validó sobre 396 deportistas de diferentes categorías competitivas $\left(\mathrm{M}_{\text {edad }}=18.74 ; \mathrm{DT}_{\text {edad }}=6.35\right)$ pertenecientes a distintas Comunidades Autónomas y disciplinas deportivas. Diversos análisis cuantitativos han permitido confirmar la bidimensionalidad de la escala $\left(\chi^{2}=183.41, \mathrm{gl}=67\right.$; $\mathrm{CFI}=.95$; TLI=.93; RMSEA=0.056-0.079; WRMR=.94), así como su adecuada fiabilidad ( $\alpha=.81$ en ambas dimensiones). Se aportan evidencias de validez en relación con otras variables como el afecto, la motivación y el apoyo a la autonomía por parte del entrenador.

Palabras clave: pasión obsesiva, pasión armoniosa, deporte, adaptación

\begin{abstract}
Passion towards an activity constitutes one of the main features when explaining both physical and emotional well-being. The type of passion experienced toward sport, obsessive or harmonious, has different effects on emotions and exercise adherence. A Spanish adaption of the Passion Scale in sport context is proposed in this paper. In the first study, the scale has been applied to 146 athletes $\left(\mathrm{M}_{\mathrm{age}}=15.94 ; \mathrm{SD}_{\mathrm{age}}=1.99\right)$. Once the scale was calibrated, 396 athletes from different competitive categories $\left(\mathrm{M}_{\mathrm{age}}=18.74 ; \mathrm{SD}_{\mathrm{age}}=6.35\right)$, Autonomous Communities and sports were assessed. Quantitative analyses confirmed the bidimensionality of the scale $\left(\chi^{2} / \mathrm{df}=2.74, \mathrm{CFI}=95\right.$; TLI=93; RMSEA=0.056-0.079; WRMR=.94), showing and adequate reliability ( $\alpha=.81$ in both dimensions). Evidence of validity in relation to other variables such as affect, motivation and coach autonomy support are provided.
\end{abstract}

Keywords: obsessive passion, harmonious passion, sport, adaption

\footnotetext{
${ }^{1}$ Universidad de Oviedo, Facultad de Psicología. Pz/ Feijóo, s/n, 33003 Oviedo, España. Tel.: 985104162. Correo: npedrosa@cop.es

${ }^{2}$ Universidad de Oviedo, Facultad de Psicología. Pz/ Feijóo, s/n, 33003 Oviedo, España. Tel.: 985104162. Correo: cueto@uniovi.es

${ }^{3}$ Universidad Pontificia Católica de Ecuador. Av. Jorge Guzmán Rueda. La Victoria. 100112. Ibarra (Imbabura), Ecuador. Tel.: 593-06 2615446. Correo: jutorrado@pucesi.edu.ec

${ }^{4}$ Universidad de Santiago de Compostela, Facultad de Psicología. Campus Sur s/n 15782 Santiago de Compostela. España. Tel.: 881813795. Correo: constantino.arce@usc.es
} 


\section{Introducción}

De manera tradicional, la Psicología ha abordado los diferentes trastornos desde una perspectiva negativa y patológica. Aunque esta visión ha generado numerosos avances (PérezÁlvarez, 2013), recientemente se ha desarrollado una línea alternativa focalizada en la intervención psicopatológica desde un enfoque positivo (Seligman \& Csikszentmihalyi, 2000).

Esta orientación, caracterizada por enfatizar el análisis de conductas relacionadas con estados positivos de las personas, ha demostrado el importante efecto potencial que variables como el afecto positivo o el optimismo presentan respecto al bienestar físico y emocional (Barrera Guzmán \& Flores Galaz, 2015; Gander, Proyer, Ruch \& Wyss, 2013; Seligman, 2008). Dentro de este conjunto de variables, la pasión experimentada hacia una actividad constituye uno de los principales aspectos a la hora de explicar el bienestar integral de la persona (Vallerand \& Verner-Filion, 2013).

La pasión se define como una fuerte inclinación de la persona hacia una actividad que considera importante en su vida, que la autodefine, le gusta y a la cuál dedica tiempo y energía (Vallerand et al, 2003). La pasión está ligada a la forma en que se practica una actividad y cómo ésta es vivida por la persona, llegando a formar parte de su identidad (Chamarro, Martos, Parrado \& Oberts, 2011). Así, se alude a una actividad apasionada cuando ésta se convierte en un elemento central de la identidad de la persona (Mageau et al., 2009).

Tomando la Teoría de la Autodeterminación (Deci \& Ryan, 1985) como marco teórico, la pasión es definida desde una perspectiva dualista. De este modo, en función del contexto en que la actividad se integre en la identidad de la persona, es posible desarrollar una pasión obsesiva o armoniosa hacia dicha actividad (Vallerand et al., 2003).

La pasión obsesiva se desarrolla cuando la participación en una tarea está regulada por presiones inter o intrapersonales, tales como el deseo de validar la autoestima, pretender la aceptación social o, incluso, debido a una falta de control sobre el sentimiento que la actividad genera (Vallerand et al., 2003). Debido a estas contingencias internas, la actividad se integra sólo parcialmente en la identidad personal (Mageau, Carpentier, \& Vallerand, 2011), pudiendo llegar a generar conflictos en otras tareas de la vida diaria (Marsh, 2013). Incluso es posible desarrollar afectos negativos y pensamientos obsesivos en torno a la actividad cuando la persona no la está practicando (Chamarro et al., 2011).

Sin embargo, la pasión armoniosa es resultado únicamente de una internalización autónoma, sin contingencias internas o externas ligadas a la actividad desarrollada (Mageau et al., 2009). De este modo, la actividad se lleva a cabo libremente, integrándola por completo en la identidad personal y propiciando con su práctica la generación de experiencias positivas, no sólo durante su práctica, sino también tras la misma (Vallerand, \& Verner-Filion, 2013).

Son múltiples las actividades que pueden generar pasión en la persona, siendo el deporte una de ellas (Chamarro et al., 2011). En el contexto deportivo, la pasión obsesiva se manifestaría cuando la persona se siente obligada a participar en su deporte, bien sea por presiones internas o externas. Por el contrario, aquellos deportistas que desarrollen una pasión armoniosa practicarán su deporte voluntariamente, mostrándose motivados hacia el mismo y generando un sentimiento de adherencia (Mageau, et al., 2011).

La pasión armoniosa se halla ligada a aspectos positivos; mientras que la pasión obsesiva se encuentra relacionada con trastornos o sintomatología psicofisiológica (e.g. Philippe, Vallerand, Houlfort, Lavigne y Donahue, 2010; Aziz, Uhrich, Wuensch, \& Swords, 2013). Estas relaciones han sido confirmadas en el ámbito deportivo, ratificando así la relevancia que esta variable presenta en este contexto (Vallerand \& Verner-Filion, 2013).

De manera sistemática, diversos estudios han mostrado una relación directa entre la pasión armoniosa y el afecto positivo, así como una percepción de apoyo del entrenador a la autonomía por parte del deportista. Un claro ejemplo es el trabajo de Lafenière, Jowett, Vallerand y Carbonneau (2011). En él se analizan 103 díadas deportista-entrenador, mostrando 
cómo aquellos entrenadores que presentan una pasión armoniosa hacia su deporte generan una relación de apoyo-autonomía adecuada, así como estados de ánimos positivos percibidos por el deportista.

Igualmente, los deportistas que presentan una pasión armoniosa muestran una motivación intrínseca a la hora de practicar el deporte, manifestando que disfrutan con la práctica deportiva y que ésta les permite satisfacer sus necesidades psicológicas básicas (Parastatidou, Doganis, Theodorakis, \& Vlachopoulos, 2012; Zazo \& Moreno-Murcia, 2015).

En contraposición, deportistas con un perfil obsesivo tienden a mostrar un afecto negativo, así como una falta de ajuste psicológico, pudiendo causar un conflicto con otras actividades cotidianas. El estudio de Vallerand, Mageau, Elliot, Dumais, Demers y Rousseau (2008, Estudio 2), el cual evalúa a 67 deportistas, muestra estas relaciones, enfatizando la función adaptativa que presenta la pasión armoniosa.

Los deportistas que desarrollan una pasión obsesiva manifiestan, además, una motivación para continuar practicando su deporte mantenida por contingencias externas e, incluso, falta de motivación para practicar su deporte, llegando a sufrir trastornos severos como el síndrome de burnout (Curran, Appleton, Hill, \& Hall, 2013; Verner-Filion, Vallerand, Donahue, Moreau, Martin, \& Mageau, 2014).

La escala desarrollada por Vallerand et al. (Passion Scale, 2003) permite llevar a cabo la evaluación de los dos tipos de pasión definidos teóricamente: obsesiva y armoniosa. Esta escala ha sido adaptada a diversos idiomas, como el chino (Lee, Liao, Huang, Shen, Wu, \& Liou,2012) o el noruego (Donahue, Forest, Vallerand, Lemyre, Crevier-Braud, \& Bergeron, 2012), entre otros; así como a múltiples contextos profesionales como el clínico, el laboral o el deportivo (e.g. Carpentier, Mageau, \& Vallerand, 2012; Lafrenière et al,. 2011; Orgambídez-Ramos, Borrego-Alés \& Gonçalves, 2014). Respecto a las propiedades psicométricas de la Escala de Pasión, ésta ha mostrado una consistencia interna adecuada, así como múltiples evidencias de validez relativas a su estructura interna, en relación a criterios externos y en relación con otras variables en diversos contextos y Revista Iberoamericana de Diagnóstico y Evaluación - e Avaliação Psicológica. RIDEP · No43 · Vol.1 · 165-176 · 2017 poblaciones (Marsh et al., 2013; Stenseng, 2008; Vallerand et al., 2003).

Como se ha mostrado, la pasión hacia la actividad deportiva es un aspecto importante que debe ser especialmente valorado en el ámbito del deporte con un objetivo doble. Por una parte, desarrollar la pasión armoniosa, pudiendo tanto potenciar la continuación en la práctica deportiva como la generación de experiencias positivas derivadas de la misma (Hodgins \& Knee, 2002). Por otra parte, intentar evitar el desarrollo de actitudes obsesivas hacia el deporte, lo cual puede derivar, si se mantiene en el tiempo, en importantes consecuencias físicas y psicológicas negativas para el deportista.

Teniendo en cuenta la relevancia que este constructo ha mostrado en el ámbito deportivo respecto a múltiples variables relacionadas con el bienestar físico y emocional, se considera necesario contar con un instrumento de evaluación que presente unas adecuadas propiedades psicométricas dirigido específicamente a esta población. El objetivo que se plantea en el presente trabajo es la adaptación de la Escala de Pasión a la población deportiva española, cubriendo una necesidad existente en este contexto. Con ello se pretende proporcionar una herramienta que permita una evaluación adecuada de este colectivo, ofreciendo así la posibilidad de definir cualquier tipo de intervención psicológica a partir de los resultados derivados de dicho instrumento.

Para ello, el trabajo se ha dividido en dos estudios. Inicialmente, se lleva a cabo un estudio piloto a partir del cual se seleccionan los ítems que formarán la escala en español. En un segundo estudio, sobre una muestra normativa, se analizan las propiedades psicométricas y las evidencias de validez del instrumento para aportar, finalmente, un baremo adaptado a la población deportiva española.

\section{Método}

\section{Participantes}

Los ítems desarrollados en el primer estudio se aplicaron a una muestra de 146 participantes entre 13 y 26 años $(M=15.94 ; D T=1.99)$, de los que el $45.90 \%$ eran hombres y el $54.10 \%$ mujeres. 
Todos ellos eran deportistas federados del Principado de Asturias que practicaban un total de ocho deportes de disciplinas tanto individuales como colectivas.

En el segundo estudio, se contó con un total de 396 participantes con edades entre 11 y 40 años $(M=18.74 ; \quad D T=6.35)$. Los participantes practicaban deporte de competición perteneciente a veinte disciplinas deportivas diferentes. De ellos, el $64.8 \%$ competía a nivel local o regional, mientras que el $35.2 \%$ restante participaba en competiciones nacionales. En cuanto a la distribución geográfica, la mayor parte de los participantes competían en la Comunidad Autónoma de Asturias (77.8\%), si bien se contó con participantes de las Comunidades de Galicia (12.1\%), Madrid (7.3\%), País Vasco (2.0\%), Andalucía $(0.5 \%)$ y Cataluña $(0.3 \%)$.

En ambos casos, se llevó a cabo un muestreo incidental.

\section{Instrumentos}

Apoyo a la autonomía por parte del entrenador. Para evaluar este constructo se aplicó el cuestionario de Clima en el Deporte (S-SCQ) en su versión española (Balaguer, Castillo, Duda, \& Tomás, 2009). El cuestionario consta de 15 ítems y aporta información sobre el nivel de autonomía proporcionado por el entrenador que el deportista percibe. En el presente estudio, el cuestionario ha mostrado una fiabilidad, estimada en términos de consistencia interna, de $\alpha=.95$.

Motivación. La Motivación ha sido evaluada mediante la adaptación española de la Escala de Motivación Deportiva (SMS; Balaguer, Castillo, \& Duda, 2007). Mediante 28 ítems, la escala ofrece información sobre el perfil motivacional del deportista, evaluando la motivación intrínseca ( $\alpha=.93)$, extrínseca $(\alpha=.83)$ y la amotivación $(\alpha=.81)$.

Afecto. El afecto de los participantes se ha evaluado mediante la versión en castellano del PANAS (Positive and Negative Affect Schedule; Robles \& Páez, 2003). La escala cuenta con 10 ítems relacionados con el afecto positivo $(\alpha=.91)$ y otros 10 ítems ligados al afecto negativo $(\alpha=.87)$.

Pasión. La pasión relacionada con el deporte se evaluó mediante la versión adaptada al castellano de la Escala de Pasión (Vallerand et al., Revista Iberoamericana de Diagnóstico y Evaluación - e Avaliação Psicológica. RIDEP · No43 · Vol.1 · 165-176 · 2017
2003), cuyo desarrollo es objetivo principal de este trabajo. La escala consta de 14 ítems que evalúan pasión obsesiva (7 ítems) y pasión armoniosa (7 ítems) mediante una escala tipo Likert de cinco alternativas.

Respecto al proceso de adaptación de la Escala de Pasión, éste se realizó mediante un proceso de retro-traducción, siguiendo las directrices internacionales para la traducción y adaptación de pruebas psicométricas (Muñiz, Elosua, \& Hambleton, 2013). Las traducciones directa e inversa fueron realizadas por grupos de especialistas en el ámbito psicométrico y deportivo, así como traductores profesionales independientes con el inglés como lengua materna. Tres de los 14 ítems mostraron inconsistencias en el proceso. En estos casos se optó por mantener dos posibles traducciones de cada ítem con objeto de seleccionar aquel que presentase un mejor funcionamiento una vez aplicado el instrumento. La adaptación inicial quedó formada por 17 ítems con un formato de respuesta tipo Likert, donde 1 implicaba un grado de desacuerdo total con el enunciado, mientras que 5 implicaba estar totalmente de acuerdo.

\section{Procedimiento}

En el primer estudio, la aplicación del instrumento fue llevada a cabo por psicólogos en los momentos previos al entrenamiento de los deportistas, de manera grupal y en una única sesión. Los clubes habilitaron el espacio para la aplicación de las pruebas. Los entrenadores y responsables de los diferentes clubes permanecieron ausentes en el momento de la aplicación con el fin de no sesgar las respuestas de los participantes.

En el segundo estudio, la aplicación de los instrumentos se llevó a cabo tanto en formato de papel y lápiz como en formato online. Las aplicaciones en papel y lápiz contaron con las mismas características que las definidas en el primer estudio. Respecto a la aplicación online, se solicitó a los responsables de los clubes deportivos que trasladasen a sus deportistas la posibilidad de colaborar en el estudio. Aquellos deportistas que accedieron a participar lo hicieron, en todos los casos, de manera privada y anónima mediante una aplicación online. 
En todos los casos se garantizó el anonimato y la confidencialidad de la prueba. La participación fue voluntaria $y$ sin recompensa alguna, mostrando los responsables de los diferentes clubes su acuerdo a que los deportistas colaborasen en el estudio.

\section{Análisis de los datos}

Una vez definida la versión traducida de la escala, en el primer estudio, se llevó a cabo un análisis de los ítems. Se estimaron los índices de discriminación de los ítems pertenecientes a cada una de las dos dimensiones. Este índice fue tenido en cuenta a la hora de seleccionar qué ítem era seleccionado en aquellos casos en que se había llevado a cabo una doble traducción. Así, el ítem con un menor poder discriminativo en cada pareja fue eliminado.

Sobre los 14 ítems finales (Anexo I) se estudió el posible Funcionamiento Diferencial de los Ítems (DIF) por sexo. Se empleó el procedimiento de regresión logística siguiendo los criterios expuestos en Gomez-Benito, Hidalgo y Zumbo (2013).

A continuación se aplicó un análisis factorial exploratorio, empleando el método de extracción de Máxima Verosimilitud y el método de rotación Promin debido a la relación esperada a priori entre ambas dimensiones (Lorenzo-Seva, 1999). La dimensionalidad de la escala se determinó empleando el método de Análisis Paralelo (Timmerman \& Lorenzo-Seva, 2011), mediante un total de 5.000 remuestreos. Finalmente se estimó la fiabilidad de cada dimensión mediante el coeficiente $\alpha$ de consistencia interna.

Sobre el grupo normativo (Estudio 2), se aplicó un análisis factorial confirmatorio que permitiese probar la estructura bidimensional de la escala. El método de extracción aplicado fue Mínimos Cuadrados Ponderados robusto (WLSMV), indicado para datos ordenados categóricamente (Elosua, 2010) y el método de rotación oblicua CF-PARSIMAX. Posteriormente se estimó nuevamente la fiabilidad del instrumento mediante el coeficiente $\alpha$ de Cronbach para datos ordinales (Elosua y Zumbo, 2008).

Se obtuvieron evidencias de validez en relación con otras variables mediante la correlación de Pearson y la correlación parcial de Revista Iberoamericana de Diagnóstico y Evaluación - e Avaliação Psicológica. RIDEP · №43 · Vol.1 · 165-176 · 2017 las dimensiones de la Escala de Pasión respecto al resto de variables evaluadas en el estudio. Al igual que en trabajos previos, se calculó la correlación parcial debido a la elevada correlación existente entre ambas dimensiones (e.g. Curran et al., 2013).

El estudio de diferencias de medias en las dimensiones de la Escala de Pasión en función del sexo, el nivel de competición (local-regional vs nacional) y la edad se llevó a cabo mediante un MANOVA con objeto de reducir los errores Tipo I. En dicho análisis se aplicó la corrección de Bonferroni para comparaciones múltiples. En el caso de la edad, se optó por agrupar a los participantes en tres grupos: sub-18, sub-23 y senior. Los tamaños del efecto para el estudio de las diferencias fueron calculados mediante el estimador eta parcial al cuadrado.

Finalmente, se establecieron los baremos en decatipos para la escala adaptada al contexto nacional.

Los diferentes análisis estadísticos han sido llevados a cabo mediante el software SPPS 19.0, MPlus 5.1 y Factor 9.2.

\section{Resultados}

\section{Estudio 1}

Mediante el índice de discriminación, se seleccionaron los ítems que presentaban un mejor funcionamiento en aquellos casos en que se había decidido mantener una doble traducción. La escala quedó así constituida por 14 ítems, siete ítems pertenecientes a cada dimensión, los cuales presentaron índices de discriminación superiores a .40 y .34 en las dimensiones de pasión obsesiva y armoniosa, respectivamente.

Respecto al estudio del DIF, ninguno de los ítems mostró funcionamiento diferencial por sexo.

El análisis factorial exploratorio permitió analizar la dimensionalidad de la escala. Los resultados mostraron la existencia de dos factores intercorrelacionados $\left(r_{\mathrm{x} 1 \times 2}=.53\right), \quad$ los cuales explicarían el $42.25 \%$ de la varianza total. Tanto los pesos factoriales como los índices de ajuste obtenidos fueron adecuados $\left(\chi^{2}=85.05, \mathrm{gl}=64\right.$, $p=.04 ; \quad$ CFI $=.94 ; \quad$ GFI $=.98 ; \quad$ TLI $=.92 ;$ RMSEA=0.048; $\quad$ RMSR $=0.054)$, pues los diferentes índices de ajuste presentan valores 
superiores a .90 y los errores residuales son inferiores a .08 (Kline, 2010). La estimación de la consistencia interna mostró coeficientes de fiabilidad de .80 y .81 para las dimensiones de pasión obsesiva y armoniosa, respectivamente.

\section{Estudio 2}

\section{Propiedades psicométricas y evidencias de validez}

A partir de los resultados obtenidos en el estudio previo se decidió aplicar, sobre la muestra normativa, un análisis factorial confirmatorio que permitiesen corroborar la bidimensionalidad del instrumento. Los índices de ajuste obtenidos fueron, nuevamente, adecuados $\left(\chi^{2}=183.41\right.$, $\mathrm{gl}=67, \quad p<.001 ; \quad \mathrm{CFI}=.95 ; \quad$ TLI=.93; RMSEA $=0.056-0.079, \quad \mathrm{NC}=90 \% ; \quad \mathrm{WRMR}=.94$; Kline, 2010).

Respecto a la fiabilidad de la escala adaptada, las dimensiones de pasión obsesiva y armoniosa mostraron, en ambos casos, coeficientes $\alpha$ de .81 .

A la hora de aportar evidencias de validez en relación con otras variables, se calcularon las correlaciones entre las dos dimensiones pertenecientes a la Escala de Pasión respecto a cada una de las dimensiones que forman las variables de motivación, estado afectivo y apoyo del entrenador a la autonomía (Tabla 1). En cada una de las celdas de la Tabla 1 se muestran dos datos: el primero de ellos muestra la correlación de Pearson, mientras que el valor entre paréntesis hace referencia a la correlación parcial una vez controlado el efecto del resto de dimensiones implicadas en cada uno de los cuatro grupos de variables (i.e. pasión, motivación, apoyo a la autonomía por parte del entrenador y afecto). Es decir, en el caso del afecto positivo, la correlación parcial muestra la relación entre afecto positivo y pasión armoniosa, controlando el influjo de la pasión obsesiva y el afecto negativo. Este procedimiento permite conocer la relación entre dos variables, eliminando estadísticamente el influjo del resto de variables consideradas. Con objeto de simplificar los resultados, únicamente se han calculado las correlaciones entre las dimensiones que han mostrado correlaciones estadísticamente significativas en trabajos precedentes $(p \leq .05)$.

\section{Estudio de diferencias}

En cuanto al estudio en ambas dimensiones de la Escala de Pasión, no se hallaron diferencias estadísticamente significativas en función del nivel de competición $(p=.086)$, si bien los deportistas de inferior nivel competitivo tienden a mostrar una mayor pasión obsesiva. Respecto a la pasión armoniosa, son quienes disputan competiciones nacionales quienes tienden a mostrar las mayores puntuaciones.

En las dos variables restantes, sexo y edad, existen diferencias estadísticamente significativas en el caso de la pasión armoniosa y obsesiva, respectivamente. En relación con el sexo, son las mujeres quienes presentan una mayor armonía con el deporte $(p=.006)$. Por contra, los hombres tienden a presentar una mayor pasión obsesiva. En función de la edad de los participantes, son los deportistas sub-18 quienes muestran los mayores niveles de pasión obsesiva, existiendo diferencias estadísticamente significativas entre este grupo respecto a los participantes de categorías sub-23 $(p=.015)$ y senior $(p=.001)$. En relación a la pasión obsesiva, existe una tendencia a que ésta se incremente a medida que aumenta la edad.

\section{Baremación de la escala}

Siguiendo el criterio de investigaciones precedentes, un deportista presentaría una elevada pasión, en cualquiera de sus subtipos, al obtener media de entre 3 y 5 puntos en la dimensión de interés (Dammyr, 2011).

Tabla 1. Correlaciones bivariadas y parciales entre la Escala de Pasión, la motivación, el afecto del deportista y el apoyo a la autonomía por parte del entrenador

\begin{tabular}{lcccccc}
\hline Pasiones & $\begin{array}{c}\text { Motivación } \\
\text { intrínseca }\end{array}$ & $\begin{array}{c}\text { Motivación } \\
\text { extrínseca }\end{array}$ & Amotivación & $\begin{array}{c}\text { Afecto } \\
\text { positivo }\end{array}$ & $\begin{array}{c}\text { Afecto } \\
\text { negativo }\end{array}$ & $\begin{array}{c}\text { Apoyo } \\
\text { autonomía }\end{array}$ \\
\hline Pasión & - & .479 & .139 & - & .309 & - \\
obsesiva & - & $(.346)$ & $(.326)$ & - & $(.287)$ & \\
Pasión & .564 & - & - & .313 & - & .262 \\
armoniosa & $(.299)$ & - & & $(.278)$ & & $(.266)$ \\
\hline
\end{tabular}


Sin embargo, en el presente trabajo se proponen baremos adaptados a la población deportiva española. A pesar de haber hallado diferencias estadísticamente significativas en el apartado precedente, éstas presentan un tamaño del efecto prácticamente nulo. Por ello, se ha optado por elaborar un único baremo conjunto para la totalidad de participantes.

Como se muestra en la Tabla 2, la zona sombreada indica la zona de riesgo en cada una de las dimensiones. Para ello, se ha tenido en cuenta la distribución de puntuaciones de cada una de las subescala en los participantes evaluados tomando, como punto de corte, la puntuación situada una desviación típica por encima y debajo de la media para la pasión obsesiva y armoniosa, respectivamente.

Tabla 2. Baremos de la adaptación española de la Escala de Pasión

\begin{tabular}{cccc}
\hline \multicolumn{3}{c}{ Pasión obsesiva } & \multicolumn{2}{r}{ Pasión armoniosa } \\
\hline PD & Decatipo & PD & Decatipo \\
\hline $7-11$ & 0 & $7-18$ & 0 \\
$12-13$ & 1 & $19-22$ & 1 \\
$14-15$ & 2 & $23-24$ & 2 \\
$16-17$ & 3 & 25 & 3 \\
$18-20$ & 4 & $26-27$ & 4 \\
$21-22$ & 5 & $28-29$ & 5 \\
$23-24$ & 6 & 30 & 6 \\
$25-27$ & 7 & 31 & 7 \\
$28-29$ & 8 & 32 & 8 \\
30 & 9 & 33 & 9 \\
$31-35$ & 10 & $34-35$ & 10 \\
\hline
\end{tabular}

Nota: $\mathrm{PD}=$ Puntuación directa

\section{Discusión}

A partir de la Teoría de la Autodeterminación (Deci \& Ryan, 1985), la cual establece que la participación en actividades que generan pasión puede entenderse como un esfuerzo por satisfacer necesidades psicológicas básicas que promueven el bienestar psicológico, la Escala de Pasión
(Vallerand et al, 2003) permite evaluar la pasión mediante dos dimensiones: obsesiva y armoniosa.

En el primer estudio se realizó un proceso de retrotraducción a partir de la escala original, desarrollando un total de 17 ítems, al haber realizado una traducción doble de tres de los ítems de la escala. Una vez comprobada su adecuada redacción, el instrumento se aplicó a una muestra piloto a partir de la cual, mediante diversos análisis cuantitativos, fueron definidos los 14 ítems finales de la versión española.

Los ítems previamente definidos fueron aplicados a una muestra más amplia de deportistas, permitiendo así analizar las propiedades psicométricas del instrumento. Dichas propiedades, al igual que en trabajos precedentes en diferentes poblaciones y contextos, resultaron adecuadas.

La consistencia interna de la escala ( $\alpha=.81$ en ambas dimensiones) mostró valores similares a los hallados en trabajos previos, en lo que ésta presenta coeficientes superiores a .75 (Vallerand y Verner-Filion, 2013).

Las evidencias de validez de la estructura interna del instrumento confirman la estructura bidimensional del mismo apuntada en múltiples trabajos precedentes (Marsh, 2013; Stenseng, 2008). Incluso, en este caso, los índices de ajuste obtenidos son ligeramente superiores a los mostrados por la escala original (Vallerand et al., 2003). Respecto a la relación con otras variables, los resultados obtenidos son prácticamente idénticos, en cuanto a magnitud y signo, a los obtenidos por trabajos anteriores en el caso del apoyo a la autonomía, la motivación intrínseca y amotivación, si bien ésta es ligeramente superior en el caso del afecto positivo (Carpentier et al., 2012; Lafrenière et al., 2011; Mageau et al, 2011; Parastatidou et al., 2012). Estos resultados permiten aportar evidencias de validez adicionales al instrumento desarrollado.

En este punto cabe destacar la importante relación existente entre la pasión obsesiva y armoniosa. La Tabla 1 confirma cómo en determinadas variables, entre las que destaca especialmente la variación obtenida en relación a la amotivación, la pasión armoniosa ejerce un importante influjo sobre la motivación (ChoongKi, Namho, \& Bo, 2014). En esta misma línea, se observa una importante variación respecto a la 
motivación intrínseca. En este caso el mayor efecto es generado por la motivación extrínseca, lo que invita a pensar sobre la existencia de reforzadores externos que, aunque secundarios, puedan afectar a la motivación y a la práctica deportiva.

Ligado a las evidencias de validez, el presente trabajo aporta un aspecto importante directamente relacionado con la estructura interna del instrumento como el estudio del DIF. En este caso, ninguno de los ítems desarrollados ha mostrado DIF respecto al sexo de los participantes, asegurando así la inexistencia de cualquier sesgo en la evaluación entre ambos grupos. Este aspecto supone una importante novedad al no haber sido analizado en trabajos precedentes, aportando así nueva información relativa a la escala.

Es importante destacar que, a pesar de haber hallado diferencias estadísticamente significativas, el tamaño del efecto se puede considerar reducido en todos los casos, por lo que dichos resultados deben tomarse con precaución debido al moderado tamaño muestral con que se cuenta. Aun así, los resultados se encuentran en la línea de trabajos previos en los que las mujeres presentan una mayor integración de la práctica deportiva en su identidad personal, mostrando los hombres una mayor obsesión por la competición y un incremento de la pasión armoniosa con la edad (e.g. Godoy, Navarrón, Vázquez, Vélez, Jiménez, \& Godoy, 2011; Kim, 2006).

\section{Conclusiones}

La adaptación realizada ha mostrado unas adecuadas propiedades psicométricas en cuanto a fiabilidad y evidencias de validez. Es posible afirmar que se cuenta con una escala breve, válida y confiable para evaluar la pasión en el ámbito deportivo. Además, se proporcionan baremos adaptados a la población española, los cuales permiten una interpretación adecuada de los resultados obtenidos en este tipo de población.

Entre las diferentes variables evaluadas, cabe destacar especialmente la relación hallada entre la pasión armoniosa y el apoyo del entrenador hacia la autonomía, por ser, en este caso, la única variable externa al propio deportista. La pasión que experimenta el deportista afecta a las relaciones interpersonales intra y extradeportivas, entre las que se encuentra la relación con el entrenador (Lafreniere, Jowett, Vallerand, Donahue, \& Lorimier, 2008). Una vez más, se confirma la importante laboral del entrenador, especialmente cuando se alude a población joven. El hecho de fomentar la autonomía de los deportistas deriva en un incremento de la pasión armoniosa, reforzando un enfoque hacia la tarea y fomentando la iniciativa del deportista, lo que puede derivar en un mayor compromiso y bienestar por parte del deportista (Borges-Silva, Prieto-Vaello, Alias, \& Moreno-Murcia, 2015).

En definitiva, resulta importante llevar a cabo evaluaciones periódicas de la pasión hacia el deporte en deportistas profesionales por dos motivos fundamentales. En primer lugar, porque el desarrollo de un perfil dominado por una pasión obsesiva conlleva, al margen de una falta de disfrute de la práctica deportiva, posibles consecuencias negativas severas si esta actitud se mantiene en el tiempo. Por otra parte porque debe tratarse de que los deportistas alcancen niveles de pasión armoniosa adecuados, especialmente, en edades jóvenes, debido a la doble función que esta variable presenta: la adherencia a la práctica deportiva y el desarrollo de beneficios emocionales para el deportista (Gómez-López, Granero-Gallegos, Baena-Extremera, \& Abraldes, 2014).

Como principal limitación del estudio es posible citar el tipo de muestreo realizado. Si bien la muestra presenta un tamaño adecuado, teniendo en cuenta la cantidad de ítems que componen el instrumento, y han participado deportistas de diferentes Comunidades Autónomas, se considera necesario aumentar tanto en número como en representatividad geográfica la muestra evaluada. Por ello, es necesario que los resultados obtenidos sean tratados con cautela, ya que pueden estar incidiendo las características inherentes a los participantes evaluados, situación que limita su generalización a la población de deportistas.

De cara a líneas futuras, resultaría interesante valorar en mayor profundidad a los deportistas de menor edad y nivel competitivo, ya que éstos destacan como aquellos que manifiestan mayores niveles de pasión obsesiva. A pesar de que habitualmente la pasión se incrementa de manera $\mathrm{N}^{\circ} 43 \cdot$ Vol.1 $165-176 \cdot 2017$ 
paralela a la edad (Chamarro et al., 2011), si estas diferencias fuesen especialmente notables, podría llegar a requerirse el abordaje de aspectos como los estilos formativos de los entrenadores, las recompensas ofrecidas a estos deportistas por competir o el entorno en que practican su deporte (Davids, 2015). Relacionado con la limitación expuesta, sería necesario replicar el estudio con una muestra de deportistas más amplia mediante un muestreo aleatorio y estratificado por Comunidad Autónoma y nivel competitivo.

\section{Referencias}

Aziz, S., Uhrich, B., Wuensch, K. L., \& Swords, B. (2013). The Workaholism Analysis Questionnaire: Emphasizing work-life imbalance and addiction in the measurement of workaholism. Journal of Behavioral and Applied Management, 14(2), 71-86

Balaguer, I., Castillo, I., \& Duda, J. L. (2007). Propiedades psicométricas de la Escala de Motivación Deportiva en deportistas españoles. Revista Mexicana de Psicología, 24, 197-207.

Balaguer, I., Castillo, I., Duda, J. L., \& Tomás, I. (2009). Análisis de las propiedades psicométricas de la versión española del Cuestionario de Clima en el Deporte. Revista de Psicología del Deporte, 18, 73-83.

Barrera Guzmán, M. L., \& Flores Galaz, M. M. (2015). Construcción de una Escala de Salud Mental Positiva para Adultos en Población Mexicana. Revista Iberoamericana de Diagnóstico y Evaluación - e Avaliação Psicológica, 39(1), 22-33.

Borges-Silva, F., Prieto-Vaello, A., Alias, A., \& Moreno-Murcia, J. A. (2015). Predicción del motivo salud en el ejercicio físico en centros de fitness. Revista Internacional de Ciencias del Deporte, 11(40), 163-172. doi:10.5232/ricyde2015.04005

Carpentier, J., Mageau, G. A., \& Vallerand, R. J. (2012). Ruminations and flow: Why do people with a more harmonious passion experience higher well-being? Journal of Happiness Studies, 13, 501-508.doi:10.1007/ s10902-011-9276-4
Chamarro, A., Martos, V., Parrado, E., \& Oberst, Ú. (2011). Aspectos psicológicos del baile: Una aproximación desde el enfoque de la pasión. Aloma: Revista de Psicologia, Ciéncies de l'Educació i de l'Esport, 29, 341350.

Choong-Ki, L., Namho, C., \& Bo, B. J. (2014). examining the structural relationships among gambling motivation, passion, and consequences of internet sports betting. Journal of gambling studies, 30(4), 845-858

Curran, T., Appleton, P. R., Hill, A. P., \& Hall, H.K. (2013). The mediating role of psychological need satisfaction in relationships between types of passion for sport and athlete burnout. Journal of Sports Sciences, 31(6), 597-606.doi:10.1080/026404 14.2012 .742956

Dammyr, M. (2011). motivation, passion and flow in norwegian sport high schools. Master thesis in Sport Sciences. Department of Coaching and Psychology: Norwegian School of Sport Sciences.

Davids, K. (2015). Athletes and sportsteams as complex adaptive system: A review of implications for learning design. Revista Internacional de Ciencias del Deporte, 11(39), 48-61.doi:10.5232/ricyde2015.03904

Deci, E. L., \& Ryan, R. M. (1985). Intrinsic motivation and self- determination in human behaviour. New York: PlenumPress

Donahue, Forest, Vallerand, Lemyre, CrevierBraud, \& Bergeron, (2012). Passion for work and emotional exhaustion: The mediating role of rumination and recovery. Applied psychology: Health and well-being, 4(3), 341368.doi:10.1111/j.1758-0854.2012.01078.x

Elosua, P. (2010). Assessing measurement equivalence in ordered-categorical data. Psicológica, 32, 403-421

Elosua, P., \& Zumbo, B.D. (2008). Reliability coefficients for ordinal response scales. Psicothema, 20(4), 896-901.

Gander, F., Proyer, R. T., Ruch, W., \&Wyss, T. (2013). Strength-based positive interventions: further evidence for their potential in enhancing well-being and alleviating depression. Journal of Happiness Studies, 14(4), 1241-1259. doi:10.1007/s10902-0129380-0. 
Godoy, D., Navarrón, E., Vázquez, M. L., Vélez, M., Jiménez, M., \& Godoy, J.F. (noviembre, 2011). Flow, competencia deportiva percibida y pasión en deportistas españoles con discapacidad según la historia de práctica deportiva y competición. Trabajo presentado en el XIII Congreso Andaluz de Psicología de la Actividad Física y el Deporte. Sevilla.

Gomez-Benito, J., Hidalgo, M. D., \& Zumbo, B.D. (2013). Effectiveness of combining statistical tests and effect sizes when using logistic discriminant function regression to detect differential item functioning for polytomous items. Educational and Psychological Measurement, 73(5), 875897.doi:10.1177/ 0013164413492419.

Gómez-López, M., Granero-Gallegos, A., BaenaExtremera A., \& Abraldes, A.J. (2014). Análisis de los perfiles motivacionales y su relación con la importancia de la educación física en secundaria. Revista Iberoamericana de Diagnóstico y Evaluación - e Avaliação Psicológica, 38(2), 11-29

Kline, R. B. (2010). Principles and practice of structural equation modeling. New York: Guilford Press

Hodgins, H. S., \& Knee, R. (2002). The integratings elf and conscious experience. En E. L. Deci y R. M. Ryan (Eds.), Handbook on self-determination research: Theoretical and applied issues (pp. 87-100). Rochester, NY: University of Rochester Press

Kim, D. (2006). Effect of sport confidence level on attributional perceptions in male \& female squash players. Journal of Coaching Development, 8(2), 125-134.

Lafrenière, M. A., Jowett, S., Vallerand, R. J., \& Carbonneau, N. (2011). Passion for coaching and the quality of the coach-athlete relationship: The mediating role of coaching behaviors. Journal of Personality and Social Psychology, 85, 756-767.doi:10.1016/j.psychs port.2010.08.002

Lafrenière, M. A. K., Jowett, S., Vallerand, R. J., Donahue, E. G., \& Lorimer, R. (2008). Passion in sport: On the quality of the coachathlete relationship. Journal of Sport \& Exercise Psychology, 30, 541-560

Lee, C. C., Liao, C. M., Huang, L. W., Shen, W. version of the Passion Scale for Sport and Exercise: Revising and structure reexamination. Journal of Sport and Exercise Psychology, 34(Suppl.), 249-249.

Lorenzo-Seva, U. (1999). Promin: A method for oblique factor rotation. Multivariate Behavioral Research, 34, 347-365

Mageau, G.A., Carpentier, J., \& Vallerand, R.J. (2011). The role of self-esteem contingencies in the distinction between obsessive and harmonious passion. European Journal of Social Psychology, 41, 720-729

Mageau, G. A., Vallerand, R. J., Charest, J., Salvy, S. J., Lacaille, N., Bouffard, T., \& Koestner, R. (2009). On the development of harmonious and obsessive passion: The role of autonomy support, activity specialization, and identification with the activity. Journal of Personality, 77(3), 601-646.doi: 10.1111/j.14 67-6494.2009.00559.x

Marsh, H. W., Vallerand, R. J., Lafrenière, M.-A. K., Parker, P., Morin, A. J. S., Carbonneau, N., ...Paquet, Y. (2013). Passion: Does one scale fit all? Construct validity of Two-Factor Passion Scale and psychometric invariance over different activities and languages. Psychological Assessment, 25(3), 796809.doi:10.1037/a0032573

Muñiz, J., Elosua, P., \& Hambleton, R. K. (2013). International test commission guidelines for test translation and adaptation: Second edition. Psicothema, 25(2), 151-157

Orgambídez-Ramos, A., Borrego-Alés, Y., \& Gonçalves, G. (2014). Adaptación y validación inicial de la versión española de la escala de pasión en el trabajo. REMA: Revista Electrónica de Metodología Aplicada, 19(2), 25-39.

Robles, R., \& Páez, F. (2003). Estudio sobre la traducción al español y las propiedades psicométricas de las escalas de afecto positivo y negativo (PANAS). Salud Mental, 26(1), 69-75.

Seligman, M. E. P. (2008). Positive health. Applied Psychology: an International Review, 57,3-18.doi:10.1111/j.14640597.2008.00351 .X.

Seligman, M. E. P., \& Csikszentmihalyi, M. (2000). Positive psychology: An introduction.

J., Wu, H. T., \& Liou, C. (2012). The chinese

Revista Iberoamericana de Diagnóstico y Evaluación - e Avaliação Psicológica. RIDEP · N43 · Vol.1 · 165-176 · 2017 
American Psychologist, 55, 5-14. doi:10.1037//0003-066X.55.1.5

Timmerman, M. E., \& Lorenzo-Seva, U. (2011). Dimensionality assessment of ordered polytomous items with parallel analysis. Psychological Methods, 16(2), 209220.doi:10.1037/a0023353

Vallerand, R. J., Blanchard, C., Mageau, G. A., Koestner, R., Ratelle, C., Léonard, M., ...Marsolais, J. (2003). Les passions de l'âme: On obsessive and harmonious passion. Journal of Personality and Social Psychology, 85(4), 756-767. doi:10.1037/0022-3514.85.4. 756

Vallerand, R. J., \& Verner-Filion, J., (2013). Making people's life most worth living: On the importance of passion for positive psychology. Terapia psicológica, 31(1), 3548.

Verner-Filion, J., Vallerand, R. J.,Donahue, E. G., Moreau, E., Martin, A., \& Mageau, G. A. (2014). Passion, coping, and anxiety in sport: The interplay between key motivational and self-regulatory processes. International Journal of Sport Psychology, 45(6), 516537.doi: 10.7352/IJSP2014.45.516

Zazo, R., \& Moreno-Murcia, J.A. (2015). Hacia el bienestar psicológico en el ejercicio físico acuático. Revista Iberoamericana de Psicología del Ejercicio y el Deporte, 10(1), 33-39. 
Anexo I. Ítems de la Escala de Pasión en su adaptación al español

\section{Pasión obsesiva}

No puedo vivir sin el deporte

Paso mucho tiempo intentando controlar mi necesidad de hacer deporte

Me cuesta imaginar mi vida sin el deporte

El deporte es casi una obsesión para mí

La necesidad de hacer deporte es tan fuerte que no puedo pensar en otra cosa

Mi estado de ánimo depende de que pueda hacer deporte

Tengo una dependencia emocional del deporte

\section{Pasión armoniosa}

El deporte está en armonía con el resto de actividades de mi vida

Estoy totalmente comprometido con el deporte

El deporte me permite vivir una gran variedad de experiencias

Las cosas nuevas que descubro con el deporte hacen que lo valore cada vez más

El deporte me permite disfrutar de experiencias inolvidables

El deporte es una pasión que aún siento que puedo controlar

El deporte refleja las cualidades que me gustan de mí 